\title{
«La terreur des esclavagistes » : la révolution française et les origines de l'abolitionnisme en Amérique
}

Battling "the Slaveholders" 'Reign of Terror" : Anti-Jacobinism and Abolitionism in the Early American Republic

\section{Rachel Hope Cleves}

\section{(2) OpenEdition}

Journals

\section{Édition électronique}

URL : https://journals.openedition.org/ahrf/11942

DOI : $10.4000 /$ ahrf.11942

ISSN : 1952-403X

Éditeur :

Armand Colin, Société des études robespierristes

\section{Édition imprimée}

Date de publication : 1 mars 2011

Pagination : 85-107

ISBN : 978-2-200-92677-9

ISSN : 0003-4436

\section{Référence électronique}

Rachel Hope Cleves, « «La terreur des esclavagistes » : la révolution française et les origines de l'abolitionnisme en Amérique », Annales historiques de la Révolution française [En ligne], 363 | janviermars 2011, mis en ligne le 01 mars 2014, consulté le 24 avril 2022. URL : http:// journals.openedition.org/ahrf/11942; DOI : https://doi.org/10.4000/ahrf.11942 


\title{
"LA TERREUR DES ESCLAVAGISTES " : LA RÉVOLUTION FRANÇAISE ET LES ORIGINES DE L'ABOLITIONNISME ENAMÉRIQUE
}

Rachel Hope CLEVES

\begin{abstract}
À la suite des massacres de Septembre, les conservateurs américains ont dénoncé toujours plus fort les « jacobins » des États-Unis. Pendant les six décennies suivantes, l'anti-Jacobinisme a ainsi souvent permis de dénoncer les mouvements démocratiques américains. On aurait tort de négliger ce discours, comme on l'a longtemps fait, au nom de son origine ancienne et de son instrumentalisation partisane. Cet article soutient au contraire que la dénonciation des effusions de sang provoquées par la Révolution française a paradoxalement nourri, jusqu'à la guerre de Sécession, une puissante opposition à la violence de l'esclavage.
\end{abstract}

Mots-clés : esclavage, jacobinisme, violence, abolitionnisme.

Pour un voyageur français aux États-Unis au cours de la période avant la guerre de Sécession, l'impact de la Révolution américaine se remarquait dans chacun des aspects de la société : dans les milliers d'organisations bénévoles auxquelles les Américains appartenaient, dans le caractère indépendant des Américaines, dans l'ardeur populaire à gagner de l'argent et dans l'égalité sociale entre hommes blancs. La culture américaine dans les années 1830 présentait un contraste frappant par rapport à celle du vieux monde liée à la tradition. Ainsi, ce fut peut-être une surprise pour Alexis de Tocqueville, lorsqu'il rencontra John Quincy Adams un soir lors de son voyage en Amérique, d'entendre l'ancien président faire des réflexions non pas sur la signification de cette révolution illustre à laquelle il avait participé, mais plutôt sur la révolution en France. Adams expliqua à Tocqueville que «les crimes de la 
Révolution française ont fait grande impression sur nous » et la nation « ressent encore ses effets $»^{1}$. L'ancien patriote vénérable, qui était entré dans le service public en tant que secrétaire diplomatique au cours de la Révolution américaine, et depuis avait servi sa nation à tous les postes possibles, y compris comme secrétaire d'État, président et membre du Congrès, pensait que la Révolution française avait transformé les ÉtatsUnis et continuait à exercer une influence très profonde quarante ans plus tard.

Lorsque Tocqueville lisait le journal au cours de son voyage, il trouvait des preuves abondantes de l'assertion d'Adams. Dans les États où Tocqueville se rendit, de la Nouvelle-Angleterre au Sud-Est et dans le Midwest, les journaux faisaient état, avec vif intérêt, des changements politiques qui se produisaient en France (tels que l'essor de la Monarchie de Juillet qui avait poussé Tocqueville à entreprendre son voyage) ${ }^{2}$. Ils remplissaient des pages de récits historiques pittoresques tirés de la Révolution française $e^{3}$. Et, de manière plus significative, ils avaient largement recours, pour exposer la politique américaine de l'époque, à un vocabulaire né de la Révolution française. Les partisans conservateurs de l'ancien président Adams s'en prenaient à son successeur démocrate Andrew Jackson, l'accusant de mener un "régime de terreur» aux États-Unis ${ }^{4}$. Les conservateurs utilisaient sans retenue le terme de « jacobins » pour attaquer les démocrates, ainsi qu'ils l'avaient fait depuis le milieu des années 1790, et ils mettaient en garde les citoyens contre des " Robespierre » potentiels chez les démocrates. Ces derniers ripostaient en qualifiant d' " aristocrates » leurs persécuteurs ${ }^{5}$. Plus tard, dans son analyse magistrale sur les États-Unis, De la démocratie en Amérique, Tocqueville fit des remarques à propos de la «violence» de la presse

(1) Alexis de Tocqueville, «Voyage en Amérique », Euvres Complètes (Journey to America, éd. J.P. Mayer, trans. George Lawrence, Londres, Faber and Faber, 1960, p. 62).

(2) «Paris on the 21st of December, 1830 », Eastern Argus Semiweekly, 18 février 1831; «Continental Affairs », Salem Gazette, 6 mai 1831. Le compte rendu de Tocqueville laisse entendre que les articles américains sur les affaires françaises informaient assez bien leurs lecteurs : «J'ai été étonné de voir combien des hommes éclairés en Amérique sont à même de débattre raisonnablement des affaires françaises » (48).

(3) «Extraordinary Escape from Death», Rhode-Island Republican, 4 avril 1831; « Polish Anecdote », Easter Argus Semiweekly, 10 juin 1831.

(4) "The ensuing will be an important election », Connecticut Courant, 29 mars 1831; " "The Reign of Terror" and Mr. Berrien », Richmond Enquirer, 28 octobre 1831.

(5) «For the Salem Gazette », Salem Gazette, 4 mars 1831; «Col. Mack », Ithaca Journal and General Advertiser, 2 novembre 1831; «Extract of a Letter from Washington », Baltimore Patriot, 11 janvier 1831; "A Pair of Noble Brothers », New-Hampshire Patriot and State Gazette, 31 janvier 1831; « See Last Page », Salem Gazette, 15 février 1831. 
politique américaine ${ }^{6}$. Il est certain que ces accusations nées pendant la Révolution française, qui s'opposaient et rebondissaient, contribuèrent à fonder son opinion.

Tocqueville choisit ses termes avec finesse lorsqu'il qualifia de « violente » la presse américaine ${ }^{7}$. Les références à la Révolution française dans les journaux américains étaient saturées de descriptions de carnages et de sang. Ainsi que l'observa John Quincy Adams, les crimes de la Révolution française avaient tout particulièrement marqué les esprits aux États-Unis, au moins de ceux qui, dans le Nord-Est, prônaient l'ordre social, comme Adams et ses alliés politiques. L'opinion des Américains à propos de la Révolution française variait entre la conviction que le siège de la Bastille avait lâché les chiens de l'enfer, et celle qu'il était le début d'un règne de mille ans de paix sur terre. Cette différence de jugement s'étendait au jacobinisme ${ }^{8}$. De nombreux penseurs démocrates, de Thomas Jefferson à ses héritiers avant la guerre de Sécession, portaient aux nues le jacobinisme considéré comme un coup triomphal en faveur des droits de l'homme. Mais les conservateurs politiques et religieux assimilaient le jacobinisme au cannibalisme et à la guillotine. Cette sorte d'anti-jacobinisme américain aurait logiquement dû décliner aux ÉtatsUnis après l'avènement de Napoléon, ou au moins après la restauration de la monarchie des Bourbons. Pourtant il se maintint de telle sorte que Tocqueville put l'observer des décennies plus tard.

La persistance de l'anti-jacobinisme aux États-Unis ne résultait pas de la survenue d'événements frappants en France même, pour intéressants qu'ils aient pu être pour des publics américains. La longévité de l'anti-jacobinisme américain découlait plutôt de l'application utile de ce discours à une politique intérieure régionale. Les conservateurs du NordEst trouvaient dans l'anti-jacobinisme un moyen puissant de lier leurs craintes d'un désordre politique démocrate à leur répugnance pour l'esclavage au Sud. De façon surprenante, l'anti-jacobinisme à l'intérieur des

(6) Alexis de Tocqueville, De la démocratie en Amérique (réf. en anglais : Democracy in America, éd. Henry Reeve et al., 2 vols., New York, Vintage Books, 1990, vol.1, p. 181-190).

(7) Dans le texte français original : «la violence de la presse ». Alexis de TocQuevilLe, De la Démocratie en Amérique, éd. Calmann Lévy, 3 vol., vol. 2, Paris, Ancienne Maison Michel Lévy Frères, 1888, p. 20.

(8) La différence de jugements des Américains sur le jacobinisme correspond, dans une certaine mesure, aux variétés d'opinions à l'intérieur même du jacobinisme français, allant de la croyance en la vertu de la terreur, à l'opposition farouche à la Terreur, à une focalisation sur la construction des mécanismes, institutions et culture d'une politique démocratique. Voir 1'historiographie jacobine récente de Carla Hesse, «The New Jacobins », French Historical Studies, 32, $\mathrm{n}^{\circ} 4,2009$. 
États-Unis devint un discours éthique et militant très fort contre l'esclavage (ceci en dépit de l'abolition de l'esclavage en 1794 par une Convention dominée par les Jacobins). Aussi longtemps que les États-Unis furent partagés entre le Nord et le Sud, quelle que soit la situation politique en France, les horreurs des Jacobins hantèrent la culture politique américaine. Même trente ans après la visite de Tocqueville, alors que les ÉtatsUnis étaient en proie à la guerre civile, le langage anti-jacobin s'invitait dans la politique américaine. À la suite de la bataille de Fort Sumter en 1861, qui marqua le début de la guerre de Sécession, le New York Herald décrivait la sécession du Sud comme un " régime de terreur » et affirmait que « les États qui ont fait sécession, nous avons quelque raison de le penser, sont tombés sous le despotisme effrayant d'un groupe de jacobins, suivis d'une cohue sur leurs talons, aussi stupides et dénués de remords que les Jacobins et la populace derrière eux dans la première Révolution française $»^{9}$. Au cours de l'événement politique déterminant pour la nation dans son premier siècle, alors que les Américains se battaient à propos de celle des institutions la plus intérieure qui soit - la possession d'esclaves en tant que biens meubles -, ils continuaient à utiliser un langage antijacobin pour décrire ce conflit.

Les conservateurs américains, particulièrement en NouvelleAngleterre, étaient fort soucieux de l'aptitude des citoyens de la nation à rester disciplinés et vertueux dès les premiers jours de la guerre de l'Indépendance. Ils s'inquiétaient que les passions humaines ne puissent pas être correctement contenues dans un système politique de volontariat. Leur inquiétude était que la Révolution avait disséminé la dépravation morale chez les citoyens américains. La fin de la Révolution n'apaisa pas ces craintes. Les désordres des années 1780, et en particulier une révolte contre l'impôt dans l'ouest du Massachusetts, connue sous le nom de "Shays' Rebellion », incita les conservateurs de Nouvelle-Angleterre à percevoir la menace imminente d'un bain de sang "anarchiste $»^{10}$.

(9) « Our Southern Rebellion », New York Herald, 20 avril 1861.

(10) David Humphreys, The Anarchiad : A New England Poem, 1786-1787, Luther G. Riggs (dir.), Gainesville, Fla., Scholars' Facsimiles \& Reprints, 1967. Également Terry Bouton, Taming Democracy: "The People," The Founders, and the Troubled Ending of the American Revolution, New York, Oxford University Press, 2007, Woody Holton, Unruly Americans and the Origins of the Constitution, New York City, Hill and Wang, 2007, Leonard L. Richards, Shay's Rebellion. The American Revolution's Final Battle, Philadelphia, University of Pennsylvania Press, 2002. 
Ces peurs contribuèrent à consolider le soutien à la Constitution dans le Nord-Est. Au cours d'une "Grande procession fédérale 》 en 1788 à Philadelphie pour fêter la ratification, ceux qui étaient en tête du défilé abattirent triomphalement deux bœufs qui, symboliquement, portaient les écriteaux 《 anarchie » et $\left\langle\right.$ confusion ${ }^{11}$. Mais les conservateurs du NordEst eurent à peine le temps de prendre une inspiration profonde avant que la nouvelle de la Révolution française n'atteigne les États-Unis et ne ressuscite le spectre de l'anarchie.

D'abord, la plupart des Américains réagirent à la Révolution française par une approbation quasi universelle, la voyant comme un miroir et un prolongement de leur propre révolution ${ }^{12}$. Quelques sceptiques, parmi lesquels John Quincy Adams et son père John Adams, exprimèrent leur vive inquiétude à propos de la violence de la Révolution française à son tout début ${ }^{13}$. Mais ils étaient largement dépassés en nombre par les optimistes qui encourageaient le gouvernement américain à en faire autant au fur et à mesure que l'État révolutionnaire en France devenait de plus en plus démocratique. Entre 1789 et 1792, les Américains consommèrent avec avidité les nouvelles venant de France, et nombreux furent ceux qui adoptèrent joyeusement des manières de parler et de s'habiller des révolutionnaires français. Ces débordements de francophilie firent frémir d'horreur les conservateurs du Nord-Est lorsque la nouvelle des massacres sanglants de Septembre franchit l'Atlantique à la fin de l'automne 1792. Les changements rapides et violents dans le gouvernement français, de même que les guerres contre ses voisins, devinrent vite les sujets principaux des articles des journaux américains ${ }^{14}$. «La France est plus folle qu'un asile d'aliénés » ainsi que l'écrivit Fisher Ames, membre fédéraliste du Congrès du Massachusetts, à un collègue politique, en octobre 1792. Dès le printemps 1793, alors que les Américains descendaient dans les rues pour fêter les victoires de la France révolutionnaire à Jemmapes et Valmy, chantaient ensemble, de manière spontanée, Ça Ira et la Marseillaise, Ames et ses alliés politiques s'inquiétaient de ce que les États-

(11) Francis Hopkinson, Account of the Grand Federal Procession, Philadelphia, July 4, 1788, Philadelphia, M. Carey, 1788.

(12) « Foreign Intelligence », Delaware Gazette, 9 septembre 1789.

(13) John Adams, Discourses on Davila. A Series of Papers, on Political History, Written in the Year 1790, and Then Published in the Gazette of the United States, Boston, Russell and Cutler, 1805; Worthington Chauncey Ford (dir.), Writings of John Quincy Adams, 3 vol., vol. 1, New York, Macmillan Company, 1913, p. 61-110.

(14) Beatrice F. Hyslop, « American Press Reports of the French Revolution, 1789-1794 », The New-York Historical Society Quarterly, XLII, nº 4, 1958. 
Unis étaient en train de suivre rapidement leur république sœur sur le chemin menant à la ruine ${ }^{15}$.

L'antipathie de la Nouvelle-Angleterre pour la Révolution française devait beaucoup à la francophobie historique dans la région, héritée du niveau élevé de participation aux guerres contre le Canada français. Constantin-François Volney fit des remarques dans ce sens après avoir résidé aux États-Unis de 1795 à 1798. Les Fédéralistes, affirmait-il, « étaient fortement teintés de ce vieux préjugé anglais et d'animosité à notre égard, état d'esprit exaspéré par les anciennes guerres du Canada $»^{16}$. La conviction très profonde des puritains concernant la tendance naturelle de l'humanité à la violence exacerbait l'horreur ressentie en NouvelleAngleterre devant les désordres politiques au cours de la Terreur. Bien que l'émergence de l'anti-jacobinisme américain puisât dans une longue tradition, il était différent du sentiment anti-français historique en ce qu'il transposait des inquiétudes à propos d'ennemis externes sur des ennemis de l'intérieur. Le 19 novembre 1792, en France, la Convention nationale vota un décret qui stipulait que la révolution républicaine était un droit universel et promettait d'aider le petit peuple de nations étrangères à gagner ses libertés ${ }^{17}$. Les Fédéralistes s'alarmaient de ce que l'expansion idéologique de la Révolution française change les démocrates américains en jacobins transnationaux qui déstabiliseraient la société civile et feraient sombrer les États-Unis naissants dans le chaos. Au cours d'une réunion politique à New York, à l'automne 1792, organisée pour discuter des événements en France, un Fédéraliste déclara que « Nous avions dans ce pays des jacobins qui cherchaient à atteindre des buts aussi pernicieux que ceux de la France, par des moyens aussi injustifiables ». Ce commentaire provoqua des protestations de la part des démocrates présents, mais de nombreux Fédéralistes acquiescèrent ${ }^{18}$. Aux États-Unis, le mot jacobin finirait par signifier n'importe quel démocrate qui semblait désireux d'utiliser des moyens violents pour parvenir à ses fins politiques.

(15) Fisher Ames à Thomas Dwight, 4 octobre 1792. Fisher AmEs, Works of Fisher Ames With a Selection from His Speeches and Correspondence, John Thornton KirkLand et Seth AmES (dir.), Boston, Little Brown and company, 1854, p. 121.

(16) «The following work, says Mr. V », The Literary Magazine, and American Register, août 1804 .

(17) Pour les réactions des Américains à ce décret, voir « From a London Paper of January 8th », The Federal Gazette, and Philadelphia Evening Post, 23 février 1793; "Foreign Affairs. London, January 9 », National Gazette, 2 mars 1793.

(18) Lettre de John Adams à Abigail Adams, 7 décembre 1792. Adams Family Papers : An Electronic Archive. Massachusetts Historical Society, http://www.masshist.org/digitaladams/ 
La circulation de l'information par tous les ports de l'Atlantique ajouta foi à la conviction des Américains que le jacobinisme était un problème qui prenait de l'importance. Les nouvelles de la Révolution française parvenaient aux États-Unis par l'intermédiaire de la presse britannique, des citoyens américains vivant en France, des capitaines de bateaux qui faisaient escale dans des ports de l'Atlantique et des Caraïbes, des réfugiés français et caraïbes qui arrivaient en grand nombre aux États-Unis, et des publications françaises envoyées depuis l'autre côté de l'océan. Ces sources variées apportaient la preuve de la violence révolutionnaire, non seulement à Paris, mais à Sheffield, Genève, Port-au-Prince, et au-delà. Les conservateurs américains étaient convaincus que le jacobinisme français ébranlait l'ordre social dans tout le monde atlantique ${ }^{19}$. Comment les États-Unis pouvaient-ils espérer échapper à une fièvre qui s'était emparée du monde?

Les sermons hebdomadaires non publiés du pasteur Elijah Parish du Massachusetts à partir des années 1790 révèlent la manière dont un habitant du Nord-Est traditionnel devint progressivement de plus en plus inquiet face au spectre du jacobinisme américain. Bien qu'en 1789 Parish eût fêté les débuts de la Révolution française, la violence en France devint sa préoccupation à l'automne 1792, en ce qu'elle était un danger pour l'ordre social local. Dans un sermon de décembre 1792, il avertit que les «partis et cabales » constituaient une menace de déstabilisation aux États-Unis à un moment où «sur le théâtre des nations vous voyez des armées qui se vautrent dans leur propre sang - la marée écarlate se répand alentour, et les rivières grossies coulent, rougies [...] vers la mer ». Parish, comme les Fédéralistes de New York qui s'étaient rassemblés un mois auparavant, entrevoyait un caractère commun, qui le terrifiait, entre les démocrates locaux et les Jacobins français. Au cours de l'année suivante, Parish tenta de s'abstenir de prêches politiques car ceux-ci perturbaient sa congrégation. Des désaccords à propos de la Révolution française provoquèrent des passions extrêmes parmi les citoyens américains, opposant

(19) «American Intelligence», The New Hampshire Spy, 28 janvier 1792; «Extract of a Letter from a Sea-Faring Man, to the under-Written, Dated Albreda, River of Gambia, June 20, 1792 », Connecticut Gazette, 10 janvier 1793; " Extract of a Letter », The Independent Gazetteer, 11 mai 1793 ; « France », Herald of the United States, 7 septembre 1793 ; « Extract of a Letter from Londonderry, Dated September 24, 1793 », The Baltimore Daily Intelligencer, 28 janvier 1794; Catherine HÉBERT, «French Publications in Philadelphia in the Age of the French Revolution A Bibliographical Essay », Pennsylvania History 58, no. 1, 1991; R. Darrell Meadows, « Engineering Exile Social Networks and the French Atlantic Community », 1789-1809, French Historical Studies 23, $\mathrm{n}^{\circ} 1,2000$. 
des frères entre eux (par exemple Fisher Ames et son frère Nathaniel se séparèrent avec acrimonie à ce sujet) ${ }^{20}$. Mais au printemps 1794 , des récits concernant la Terreur amenèrent Parish à critiquer à nouveau les « cruautés inouïes » à la suite desquelles le «sol [de France] avait été inondé de sang » et qui menaçaient de provoquer une "pluie de sang » qui viendrait "rougir nos champs». Dès 1795, Parish fut localement connu comme un anti-jacobin engagé. À la fin de la décennie, Parish se mit à publier des sermons pour une large distribution qui attaquaient le jacobinisme à l'étranger et dans le pays. Au cours des trois décennies suivantes, le sentiment anti-jacobin des Américains s'exprima, avec vigueur, par sa voix ${ }^{21}$.

Les écrits de Parish, typiques de la focalisation gothique des antijacobins américains, portaient sur le sang qui coulait, les champs parsemés de cadavres et les corps mutilés. La persistance des images de carnages jacobins aux États-Unis au XIX ${ }^{\mathrm{e}}$ siècle est en partie due à la nouveauté et à la profusion du langage qui les décrivait. De 1790 à 1815 , les États-Unis furent bombardés de textes dénonçant la Révolution française et ses séquelles napoléoniennes. Ces récits «pénétrèrent dans toutes les fermes », selon un commentateur conservateur en 1799, et « ils n'en sortiront pas, jusqu'à ce que des histoires de tomahawks indiens et de danses de la guerre autour de prisonniers les remplacent $\gg^{22}$. Nombreux furent les récits anti-jacobins qui donnaient des descriptions précises de tortures et de brutalités et utilisaient un vocabulaire affreusement explicite. La menace intérieure que constituait le jacobinisme transatlantique inquiétait tant les conservateurs américains qu'ils avaient besoin que se crée un nouveau langage, un mode américain d'écriture du gothique, afin de tenir compte de ses énormités. Le Fédéraliste du Connecticut John Cotton Smith affirma, dans un discours le 4 juillet 1798, qu' ' il faut inventer de nouveaux mots, de nouveaux sons, s'ils doivent jamais relater, autant que faire se peut, toutes les atrocités sans précédent qui ont marqué le déroulement de la Révolution française ». Les événements de la Révolution

(20) Charles Warren (dir.), Jacobin and Junto, or Early American Politics as Viewed in the Diary of Dr. Nathaniel Ames, 1758-1822, Cambridge, Harvard University Press, 1931.

(21) Elijah PARISH, «Micah $2.10 »$, décembre 1792; «Ezra 8.23 », avril 1794; Elijah Parish Papers, Governor's Academy; Elijah PARIsh, An Oration Delivered at Byfield July 4, 1799 , Newburyport, Mass., Angier March, 1799.

(22) Cité par Vernon STAUfFER, New England and the Bavarian Illuminati, vol. LXXXII, $\mathrm{n}^{\circ}$ 1, Columbia University. Studies in History, Economic, and Public Law, New York, Columbia University Press, 1918, p. 127. John Cоттоn Sмітн, An Oration, Pronounced at Sharon, on the Anniversary of American Independence, 4th July, 1798, Litchfield Conn., T. Collier, 1798, p. 5. 
française étaient « écrits en lignes de sang ». Smith assura que les récits de ces horreurs devaient aussi être transcrits de la même manière ${ }^{23}$.

Les textes anti-jacobins en Amérique abondaient en images d'effusions de sang. Les mots "sang », " sanglant», "assoiffé de sang » et « carnage » revenaient sans cesse. L'anti-jacobinisme puisait dans une longue histoire de rhétorique anglaise qui utilisait le sang en tant que symbole de force illégitime ou de cruautée ${ }^{24}$. Les auteurs anti-jacobins montraient souvent le sang qui coulait sans s'arrêter, suggérant que la brutalité qu'il représentait, une fois déclenchée, ne pouvait pas être maîtrisée. La violence engloutirait tout système politique qui libérerait son potentiel de destruction. De nombreux pasteurs et orateurs dépeignaient la France comme étant inondée par des «torrents de sang $»^{25}$. D'autres faisaient état de « ruisseaux de sang humain » ou de « rivières de sang $»^{26}$. Ce qui se passait en France était évoqué par des expressions comme « spectacle ensanglanté $»$ ou « scènes sanglantes $»^{27}$. Pour varier, les anti-jacobins qualifiaient souvent la révolution de « sanguinaire $»^{28}$. De nombreuses sources anti-jacobines américaines reprenaient une autre formulation, celle de " vêtements roulés dans le sang » (Isaïe 9:5); cette image est utilisée pour décrire les horreurs de la guerre, et fait surgir à l'esprit un champ de bataille si imprégné de sang que les victimes se vautrent littéralement dedans ${ }^{29}$.

(23) John Cotton Smith, An Oration, Pronounced at Sharon, on the Anniversary of American Independence, 4th July, 1798, op. cit.

(24) Philippe Rosenberg, The Moral Order of Violence The Meanings of Cruelty in Early Modern England, 1648-1685, thèse de doctorat, Duke, 1999, chapitre 3.

(25) Jonathan Freeman, A Sermon Delivered at New-Windsor and Bethlehem, August 30, 1798, New-Windsor N.Y., Jacob Schultz, 1799; Samuel Kendal, A Sermon Delivered on the Day of National Thanksgiving, February 19, 1795, Boston, Hall, 1795, p. 11; William LinN, A Discourse on National Sins Delivered May 9, 1798; Being the Day Recommended by the President of the United States to Be Observed as a Day of General Fast, New York, T.\&J. Swords, 1798; David TAPPAN, Christian Thankfulness Explained and Enforced A Sermon Delivered at Charlestown, in the Afternoon of February 19, 1795, Boston, Samuel Hall, 1795, p. 27.

(26) Timothy Dwight, The Duty of Americans at the Present Crisis Illustrated in a Discourse, Preached on the Fourth of July, 1798, New Haven, Thomas and Samuel Green, 1798, David Osgood, The Devil Let Loose, or the Wo Occasioned to the Inhabitants of the Earth by His Wrathful Appearance among Them Illustrated in a Discourse Delivered on the Day of the National Fast, April 25, 1799, Boston, Samuel Hall, 1799.

(27) « State of French Politicks », Impartial Herald, 13 janvier 1795 ; « Letter of General Dumourier », Columbian Centinel, 15 mai 1793; « New York », Commercial Advertiser, 9 octobre 1797.

(28) An Impartial History of the Late Revolution in France, from Its Commencement to the Death of the Queen, and the Execution of the Deputies of the Gironde Party, vol. 1, Philadelphia, M. Carey, 1794, p. 79.

(29) John Andrews, A Sermon, Delivered February 19, 1795 Being a Day of Public Thanksgiving, Throughout the United States of America, Newburyport, Mass, Blunt \& March, 1795, 
Au fil des années, les textes anti-jacobins devinrent de plus en plus frappants dans leurs détails. Au lieu de pâlir comme de vieilles photographies, leurs couleurs, comme par contradiction, s'intensifièrent. Alors que les critiques envers la Révolution faites pendant la Terreur reprenaient succinctement en quelques paragraphes les carnages et assassinats, les textes écrits après 1795 ne pouvaient contenir tout le sang; ils se transformèrent en catalogues d'horreurs qui se poursuivaient sur des pages et par chapitres entiers. William Cobbett, expatrié anglais vivant à Philadelphie, perfectionna le gothique anti-jacobin américain dans son livre The Bloody Buoy écrit en 1796. Ce texte, qui connut de nombreuses éditions en Amérique, fut réimprimé et fort bien reçu en Grande-Bretagne, ce qui illustra les liens étroits entre les anti-jacobins américains et britanniques dans les années $1790^{30}$. Délaissant la narration, Cobbett organisa The Bloody Buoy pour en faire une énumération des horreurs puisées dans des sources françaises. Son trope principal était le cannibalisme. Les exemples d'anthropophagie cités dans la table des matières comprenaient les suivants : " un homme arrache le cœur tout chaud d'une femme et le déchire de ses dents »; «Femmes rôties vivantes, leur chair découpée et offerte comme nourriture aux hommes »; « une femme étendue, morte, un enfant tétant son sein »; « un homme en invite un autre à goûter la cervelle d'un aristocrate »; " Goullin bat son propre père sur son lit de mort, et dit qu'aucun homme ne devrait être considéré comme bon révolutionnaire s'il n'a pas le courage de boire un verre de sang humain ». D'autres tropes récurrents dans ce livre incluaient la mutilation de cadavres et le massacre de petits enfants. Ces faits horribles étaient décrits avec des détails évocateurs. Un passage illustrant le meurtre de prêtres attira précisément l'attention sur la manière dont « le dallage de marbre était couvert d'ordures, de sang et de carcasses [sic] mutilées, et les côtés de l'autel éclaboussés de sang et de cervelle $\gg{ }^{31}$.

Ce qui effraya le plus les anti-jacobins américains à propos de la Révolution française fut la rapidité avec laquelle les Français avaient,

9, Ezra SAmpson, A Discourse Delivered February 19, 1795 Being the Day of National Thanksgiving, Boston, Printed and sold by Samuel Hall, $\mathrm{n}^{\circ}$ 53, Cornhill Boston., 1795; Thomas Cushing ThACHER, A Sermon. Preached at Lynn, November 20 $0^{\text {th }} 1794$ Being the Day Appointed for the Annual Thanksgiving, Printed at Boston, Thomas Hall, 1794.

(30) Rachel Hope Cleves, "Jacobins in This Country": The United States, Great Britain and Trans-Atlantic Anti-Jacobinism », Early American Studies 8, n 2, 2010.

(31) William CoвBetт, The Bloody Buoy Thrown out as a Warning to the Political Pilots of All Nations : Or a Faithful Relation of a Multitude of Acts of Horrid Barbarity, Such as the Eye Never Witnessed, the Tongue Never Expressed, or the Imagination Conceived, until the Commencement of the French Revolution, Philadelphia, Cobbett, 1796. 
semble-t-il, dégénéré pour devenir des bêtes féroces et des démons. Les Américains croyaient que la France avait occupé le pinacle de la civilisation avant la Révolution. La soudaineté avec laquelle la nation était censée s'être abaissée à la violence cannibale laissait présager qu'un sort similaire, ou pire, planait sur tout peuple moins parfait et assez fou pour adopter les mêmes principes perturbateurs. "N'est-il pas étonnant de voir comment un si grand changement dans la moralité et les manières d'une nation puisse s'effectuer si soudainement »? (pour reprendre la question posée par le pasteur du Massachusetts David Osgood à ses fidèles lors de son sermon de "Thanksgiving » en 1794) " $^{32}$. Cobbett fit de même la remarque que la Révolution française « changeait les Français éthérés pour en faire un groupe monstrueux composé des chiens courants les plus féroces $»^{33}$. Si les démocrates américains devenaient des jacobins, Osgood et Cobbett sous-entendaient qu'ils subiraient la même transformation.

Les chefs de file conservateurs qualifiaient ce déclin précipité de processus « dé-civilisateur». En abolissant les contraintes de la loi et de la religion, la Révolution française avait ôté aux Français leur sens moral et en avait fait des barbares. Le dénigrement vis-à-vis du christianisme et le rejet de toute déférence à l'égard des autorités civiles ou religieuses eurent pour effet que les Français cessèrent d'obéir aux injonctions morales qui contenaient la violence des hommes. Attaquer la religion donnait libre cours à la dépravation naturelle des Français. Des politiciens démagogues et des hommes ambitieux manipulaient alors facilement ceux qui avaient été brutalisés pour leur faire commettre des actes de violence contre des opposants politiques et d'autres victimes malchanceuses. La république devenait la proie de factions rivales et de la guerre civile. Dans le cadre d'une guerre totale, toute autorité morale se désagrégeait et les Français retournaient à leur état dépravé d'humanité non rachetée ${ }^{34}$.

En dépit des récits anti-jacobins sur les nombreuses horreurs commises au cours de la Révolution française, les progressistes américains continuèrent à défendre la Révolution française tout au long des

(32) David Osgood, The Wonderful Works of God Are to Be Remembered. A Sermon, Delivered on the Day of Annual Thanksgiving, November, 20, 1794, Boston, Samuel Hall, 1794, p. 23.

(33) William CoBbetr, [Pierre le Porc-Epic] Porcupine's Works, London, Cobbett and Morgan, 1801

(34) John M. Mason, Mercy Remembered in Wrath A Sermon, the Substance of Which Was Preached on the 19th of February, 1795, New-York, J. Buel, 1795, p. 20. 
années 1790 et à exiger davantage de démocratie dans leur pays. Le secrétaire d'État Thomas Jefferson réagit de façon détestable aux nouvelles de violences à Paris au cours de l'hiver 1792 en demandant s'il y avait « jamais eu une telle récompense gagnée avec si peu de sang innocent $» ?^{35}$ Alors que les progressistes en politique aux États-Unis s'unirent sous la houlette de Jefferson pour former un nouveau parti démocrate, ils restèrent aussi fidèles à la Révolution, défendant même la Terreur. Le démocrate de New York, James Westcott, fanfaronna, en 1794, déclarant que la France «n'hésiterait pas à faire monter ses fumées avec le sang sacrilège de ces misérables scélérats qui violent son mandat et font la guerre à ses fervents admirateurs $»^{36}$. De telles louanges à propos des exécutions collectives en France renforçaient les craintes des conservateurs à l'égard du jacobinisme américain.

Deux insurrections à l'intérieur des États-Unis dans les années 1790 donnèrent aux conservateurs américains des raisons supplémentaires de s'inquiéter. Au cours de l'été 1794, des fermiers dans l'ouest de la Pennsylvanie eurent recours à des tactiques violentes pour protester contre la taxe sur le whisky du gouvernement fédéral. Un des meneurs de la rébellion, David Bradford, menaça d'établir un comité de salut public et de commencer à construire des guillotines ${ }^{37}$. L'ambassadeur de France aux États-Unis, Jean Antoine Joseph Fauchet, approuva publiquement le soulèvement ${ }^{38}$. Alexander Hamilton, figure montante à la tête du parti fédéraliste et anti-jacobin déclaré, mena une armée vers l'ouest de la Pennsylvanie pour réprimer la révolte (qui cessa sans grande effusion de sang). Mais, cinq ans plus tard, la résistance armée en Pennsylvanie contre un impôt national pour financer la guerre des États-Unis contre la France convainquit les Fédéralistes que la menace d'un jacobinisme transatlantique restait forte. Certains des participants à la « Fries Rebellion » de 1799 portaient des bonnets rouges français ou

(35) Thomas Jefferson à William Short, 3 janvier 1793, Thomas Jefferson, The Papers of Thomas Jefferson, Charles T. Cullen, John Catanzariti et Julian P. Boyd (dir.), Princeton, N.J., Princeton University Press, 1950, vol. 25, p. 14.

(36) James D. Westcott, An Oration, Commemorative of the Declaration of American Independence Delivered before the Ciceronian Society, on the Fourth of July, M, DCC, XCIV; and Published at Their Request, Philadelphia, William Young, 1794, p. 13.

(37) «Lewiston, (Mifflin County) July 5th, 1794 », Kline’s Carlisle Weekly Gazette, 9 juillet 1794. Les anti-jacobins savaient que les insurgés menaçaient de construire une guillotine; voir David Osgood, A Discourse, Delivered February 19, 1795 The Day Set Apart by the President for a General Thanksgiving through the United States, Boston, Samuel Hall, 1795, p. 23.

(38) Paul Douglas Newman, «The Federalists' Cold War The Fries Rebellion, National Security, and the State, 1787-1800», Pennsylvania History 67, n 1, 2000, p. 74. 
des cocardes et faisaient ouvertement état de leur communauté de vues avec la cause transatlantique ${ }^{39}$. Deux des insurgés menacèrent d'aller de maison en maison et de « couper la tête » des collecteurs d'impôts ${ }^{40}$. Alors que les révoltes démocratiques contre l'impôt avaient une longue histoire dans le pays, sans rapport avec la France, les allusions sans équivoque à la Révolution française que faisaient les rebelles confirmaient les craintes des Fédéralistes que le jacobinisme se répandait dans le pays.

Si ce qui se passait en France était la plus grande raison de s'alarmer pour les anti-jacobins américains, alors la fin du régime de terreur, ou au moins le coup d'État de Napoléon en 1799, aurait pu ramener la sérénité aux États-Unis. Mais les conservateurs du Nord-Est craignaient les «jacobins» de l'intérieur davantage que ceux de l'étranger et par conséquent l'anti-jacobinisme persista aux États-Unis en tant que discours politique local bien après qu'il eut cessé d'être pertinent ailleurs. Les conservateurs américains créèrent un jargon anti-jacobin qui utilisait généreusement des termes comme « jacobin » et " régime de terreur », d'une manière telle qu'elle irritait à coup sûr quiconque était bien au courant du sens précis de ces expressions en France. Lorsqu'ils évoquaient les affaires étrangères, les conservateurs américains usaient souvent du terme de «jacobin » pour faire référence à n'importe qui associé à la Révolution française. Lorsqu'il était question des affaires locales, les Fédéralistes et les pasteurs traditionnels se servaient du mot « jacobin » pour évoquer les démocrates qui avaient recours à la violence (émeutes, attaques d'officiels, paroles menaçantes) pour arriver à leurs fins politiques. Dans cette dernière catégorie, les propriétaires d'esclaves du Sud, qui, à ce qu'on disait, étaient enclins à la violence, devinrent une cible favorite.

Des écrits allégoriques et allusifs qui établissaient un lien entre la violence jacobine et celle des esclavagistes commencèrent à paraître au début des années 1790. Les Fédéralistes faisaient correspondre les deux du fait de la puissance du parti démocrate « jacobin » dans le Sud (ses dirigeants étaient des Virginiens tels Thomas Jefferson et James Madison). Les anti-jacobins du Nord-Est affirmaient que la possession d'esclaves noirs rendait le recours à la violence habituel parmi les dirigeants du Sud

(39) Simon Newman, «The World Turned Upside Down Revolutionary Politics, Fries` and Gabriel's Rebellions, and the Fears of the Federalists », Pennsylvania History 67, $\mathrm{n}^{\circ}$ 1, 2000.

(40) Robert H. Churchill, «Popular Nullification, Fries Rebellion, and the Waning of Radical Republicanism, 1798-1801 », Pennsylvania History 67, n 1, 2000, p. 118. 
et faisait qu'ils étaient fort disposés à soutenir à la fois et la violence des Jacobins français, et celle des démocrates aux États-Unis. Par exemple un Fédéraliste du Massachusetts, Christopher Gore, écrivit un ensemble d'essais sous le pseudonyme de «Manlius » en 1794, qui représentaient les sudistes comme " dépendant du travail des esclaves », ce qui les rendaient « forcément en faveur de la domination », de même qu'ils étaient " amis de la Révolution française » pleins de « haine pour [leur] propre pays » et du désir de « se rebeller et mener une insurrection ouverte ${ }^{41}$. Alors que les tensions politiques et locales persistèrent jusqu'au début $\mathrm{du} \mathrm{XIX}^{\mathrm{e}}$ siècle, les accusations de jacobinisme esclavagiste devinrent plus explicites. Par exemple, en 1815, le New-Yorkais Jarvis Brewster, exprima le grief que l'esclavage était « un système de tyrannie et de persécution plus horrible peut-être que celui mis en pratique par un Néron ou un Robespierre ${ }^{42}$. Au cours de la période précédant la guerre de Sécession, les tropes anti-jacobins étaient largement répandus dans les écrits abolitionnistes. Des articles de journaux, comme « "La Terreur" des esclavagistes ", imprimé en 1837, dépeignaient les propriétaires d'esclaves comme des jacobins qui, tels des pillards, volaient les « os et les muscles, la chair et le sang » de leurs esclaves ${ }^{43}$. Ces remarques conduisirent finalement à l'assertion du New York Herald en 1861 que les sécessionnistes du Sud étaient des jacobins. L'attaque du Herald avait du sens dans l'esprit de générations d'habitants du Nord élevés au son du discours public qui, de manière habituelle, comparait les esclavagistes à Robespierre.

L'antiesclavagisme anti-jacobin trouvait ses racines dans les attaques portées dans le Nord-Est contre l'esclavage au cours de la Révolution américaine. De nombreux pasteurs congrégationalistes conservateurs avaient représenté la Révolution américaine comme une punition divine pour l'esclavage, crime national ${ }^{44}$. Pendant la Révolution amé-

(41) Christopher Gore, Manlius With Notes and References, Boston, Benjamin Russell, 1794 , p. 49, p. 51, p. 53.

(42) Jarvis Brewster, An Exposition of the Treatment of Slaves in the Southern States, Particularly in the States of Maryland, Virginia, North Carolina, South Carolina and Georgia, New Brunswick [N.Y.], D.\& J. Fitz Randolph, 1815, p. 7.

(43) "The Slaveholders" "Reign of Terror" », Philadelphia National Enquirer, 29 avril 1837.

(44) David Brion Davis, The Problem of Slavery in the Age of Revolution, 1770-1823, Ithaca, Cornell University Press, 1975, chapitre 7; Peter Hinks, « Timothy Dwight, Congregationalism, and Early Anti-Slavery », dans The Problem of Evil Slavery, Freedom, and the Ambiguities of American Reform, Steven Mintz et John Stauffer (dir.), Amherst, University of Massachusetts Press, 2007; Kenneth P. Minkema et Harry S. Stout, «The Edwardsean Tradition and the Antislavery Debate, 1740-1865 », Journal of American History 92, n 1, 2005. 
ricaine, les dirigeants politiques du Nord-Est contestèrent également l'institution de l'esclavage, mettant en branle son abolition progressive partout au nord du Delaware au début du siècle suivant ${ }^{45}$. Ceux qui, dans le Nord-Est, militaient le plus intensément pour une abolition prochaine étaient inspirés moins par l'esprit démocratique de la Révolution que par la crainte que l'esclavage fût une institution porteuse de violence sociale qui constituait une menace pour une liberté bien ordonnée. Tout comme la peur de désordres sociaux au cours des années 1780 avait incité les conservateurs du Nord-Est à réagir fébrilement à la menace du jacobinisme américain, les inquiétudes de la période révolutionnaire à propos de l'indiscipline des propriétaires d'esclaves disposèrent les conservateurs à voir un lien entre le jacobinisme américain et l'esclavage.

Les critiques fédéralistes qui, au moyen d'une allégorie, liaient l'esclavagisme au Sud et les jacobins, gagnèrent en crédibilité par rapport à la politique de la rue utilisée par les démocrates jeffersoniens dans les années 1790. En 1795, les démocrates s'opposèrent au traité Jay entre les Anglais et les Américains, négocié par les Fédéralistes : des émeutes éclatèrent dans plusieurs villes y compris Charleston, Savannah, Baltimore, Philadelphie, New York et Boston ${ }^{46}$. Les démocrates du Sud vilipendaient le traité Jay, non seulement parce qu'il mettait fin à tout espoir d'une alliance américaine avec la France, mais parce qu'il n'obligeait pas la Grande-Bretagne à rémunérer les habitants du Sud pour les esclaves qu'elle avait « emportés » à la fin de la Révolution. Des manifestations au cours desquelles des démocrates du Sud guillotinèrent des effigies du Fédéraliste John Jay persuadèrent les Fédéralistes du Nord-Est qu'il existait une relation entre le jacobinisme dans le Sud et l'esclavage ${ }^{47}$. Le concept du processus dé-civilisateur combla le fossé entre la violence des esclavagistes et celle des démocrates en révélant que toutes deux provenaient d'une origine commune : l'échec de l'individu à contrôler ses passions dépravées et à obéir à une autorité morale. Thomas Jefferson lui-même avait admis que l'esclavage conduisait les maîtres à « la mise en

(45) Arthur Zilversmit, The First Emancipation; the Abolition of Slavery in the North, Chicago, University Press, 1967.

(46) Paul GiLJE, Liberty on the Waterfront American Maritime Culture in the Age of Revolution, Daniel K. Richter et Kathleen M. Brown (dir.), Early American Studies, Philadelphia, University of Pennsylvania Press, 2004, p. 143.

(47) Connecticut Courant, 3 août 1795; Henry WiLson, History of the Rise and Fall of the Slave Power in America, New York, Negro Universities Press, 1969, chapitre 9. 
œuvre perpétuelle des passions les plus tumultueuses $»^{48}$. Cette impulsion faisait des esclavagistes du Sud des jacobins naturels.

Thomas Jefferson subit les attaques antiesclavagistes et antijacobines les plus injurieuses dans les débuts de la période nationale, souvent en des termes provenant de ses propres écrits. Les offensives contre Jefferson en tant qu'esclavagiste jacobin accaparaient les opposants à sa campagne présidentielle en 1800. Dans les essais Jeffersoniad publiés au cours de l'été 1800, l'auteur anonyme prévenait que les buts de Jefferson étaient de « jacobiniser, révolutionner le peuple de ces États-Unis, et, bien sûr, lui faire perdre tout sens moral ». Ces essais accusaient Jefferson d'être athée, charge qui connotait la tache de violence dépravée - puisque l'athéisme en France avait donné lieu à des «actes de la férocité la plus extrême ». Ces essais condamnaient aussi Jefferson pour être à la tête d'un parti « de propriétaires d'enfants bâtards » « d'affameurs d'enfants bâtards », « de voleurs de Nègres » et " de tueurs d'hommes », parti qui " grince des dents de rage et ressent une soif que rien n'étanchera, sinon le sang de proscriptions ». Pendant trois mois, les Jeffersoniad se répandirent sur les dangers que le candidat présidentiel présentait pour la république. S'il était élu à la magistrature suprême de la nation, « rien » ne se passerait en Amérique « si ce n'est la reprise des scènes sanglantes qui affligèrent tant la grande ville de Lyon, pendant le règne de Robespierre $»^{49}$. L'antijacobinisme et l'antiesclavagisme se combinèrent dans les Jeffersoniad pour fournir un langage de parti pris et d'insulte.

Au fil des années, le rattachement de la violence des jacobins à la brutalité de l'esclavage devint également monnaie courante dans des textes impartiaux. Les abolitionnistes découvrirent que le mode d'écriture gothique perfectionné dans la lutte contre la Révolution française fonctionnait très bien dans le combat zélé contre l'esclavage. En 1795, une convention nationale contre l'esclavage, tenue à Philadelphie, annonça la décision des abolitionnistes de concentrer leur attention sur la publication de récits gothiques de violences esclavagistes, plutôt que de tabler sur des arguments de droits naturels. L'esclavage en Amérique avait survécu jusque-là, selon la conclusion de la convention, du fait d' « un manque de réflexion ». Les Américains, particulièrement les propriétaires d'esclaves,

(48) Thomas Jefferson, Notes on the State of Virginia, William Harwood Peden (dir.), Chapel Hill, Published for the Institute of Early American History and Culture Williamsburg Va. by the University of North Carolina Press, 1954, p. 162. [Notes sur L'État de Virginie]

(49) «The Jeffersoniad» ${ }^{o s}$. I - XVI, dans The Columbian Centinel and Massachusetts Federalist, juin-août 1800. Les essais furent repris par The Newburyport Herald, The Gazette of the United States, et The Philadelphia Gazette, entre autres journaux. 
devaient être réveillés grâce à " la persuasion de l'éloquence » pour être saisis « d'horreur devant l'énormité de leur conduite $»^{50}$. Après 1795 , les auteurs antiesclavagistes mirent de plus en plus l'accent sur la brutalité qui définissait l'esclavage, au lieu d'insister simplement sur son injustice. Ces critiques utilisèrent un grand nombre des tropes mêmes qui figuraient si abondamment dans les écrits anti-français. Les écrivains antiesclavagistes utilisèrent le sang comme symbole récurrent au cours de leurs attaques contre cette institution, évoquant sa couleur, sa viscosité et son abondance. Ils cataloguèrent les propriétaires d'esclaves de cannibales et décrivirent le Sud esclavagiste comme un paysage de mort ${ }^{51}$. Les auteurs antiesclavagistes finirent par faire directement appel au langage de l'antijacobinisme pour présenter leurs arguments.

Reflections on the Inconsistency of Man, Particularly Exemplified in the Practice of Slavery (1796), d'Amynto, est l'un des premiers textes américains antiesclavagistes à accuser explicitement les propriétaires d'esclaves d'être de violents jacobins. Cette brochure anonyme, publiée à New York par un imprimeur conservateur d'ouvrages anti-jacobins, plaidait pour l'« abolition totale » et définissait l'esclavage comme une institution de la violence causée par la même dépravation de l'homme que celle qui inspirait les guerres et le meurtre. En outre, Amynto qualifiait les esclavagistes de jacobins violents, affirmant que parmi les « représentants [...] [des États] du Sud au Congrès, où l'esclavage continue à se déchaîner dans toutes ses horreurs, une démocratie furieuse, copiée sur les principes jacobins français, semble être le souhait de la plupart des membres de la bonne société du Sud ». Amynto ajoutait foi à ces assertions de violence esclavagiste en attirant l'attention sur le soutien que la bonne société du Sud apportait à la Révolution française. «Il n'est pas inhabituel» selon Amynto «de voir des enfants armés de fouets et de triques, frapper et brutaliser de pauvres vieux Nègres décrépits », alors que des hommes violaient leurs esclaves et vendaient leurs propres enfants. Les lecteurs pouvaient être tentés de ne pas retenir de telles histoires extraordinaires

(50) American Convention, Minutes of the Proceedings of the Second Convention of Delegates from the Abolition Societies Established in Different Parts of the United States Assembled at Philadelphia, Philadelphia, Zachariah Poulson Junr, 1795.

(51) Winthrop JoRdan, White over Black American Attitudes toward the Negro, 1550-1812, Chapel Hill, Omohundro Institute of Early American History and Culture, University of North Carolina Press, 1968, p. 367-371, Kimberly K. Sмгтн, The Dominion of Voice : Riot, Reason, and Romance in Antebellum Politics, Lawrence, University Press of Kansas, 1999; Lorenzo Dow TuRner, Anti-Slavery Sentiment in American Literature Prior to 1865, Washington, D.C., The Association for the Study of Negro Life and History, 1929, chapitre 1. 
d'enfants torturant des vieillards et de parents vendant leurs enfants, mais parce qu'il mettait sur le même plan esclavagistes et Jacobins, Amynto rendait ses charges contre l'esclavage plus persuasives.

Les Reflections on the Inconsistency of Man sous-entendaient que les Jacobins et les propriétaires d'esclaves étaient semblables parce qu'aucun des deux groupes ne soumettrait son pouvoir à des restrictions légales ou morales. Les esclavagistes étaient si accoutumés à détenir une autorité absolue, même au point de tuer les êtres humains en leur possession, qu'ils ne pouvaient contenir leurs passions dans la sphère politique. Le sang versé par le fouet du maître d'esclaves et celui répandu par la guillotine coulaient vers une mare commune. Dans les deux pays, «la voix de sangs fraternels s'élève du sol et crie vers moi ». La délivrance de ces deux cauchemars ne pourrait venir que de la libération des hommes de l'asservissement civil et moral :

« Si nous regrettons sincèrement ces scènes horribles de violence qui ont été perpétrées par ces scélérats et coupe-gorge qui ont ravagé la France; si nous compatissons devant l'histoire tragique de ces multitudes de vies innocentes qui ont versé leur sang sous l'atroce lame de la guillotine $[\ldots]$ [nous] espérons que les révolutions d'Amérique et de France ne sont que les mouvements préalables vers une émancipation générale de la nature humaine $»^{52}$.

Les lecteurs de textes anti-jacobins qui avaient rejeté la Révolution française après que ses « scènes horribles de violence » eurent été exposées, furent obligés, par la même logique, de repousser les horreurs de l'esclavage en Amérique et de prier pour une " émancipation générale ». Les Reflections se servirent du langage de l'anti-jacobinisme pour résumer l'attitude de refus des lecteurs vis-à-vis de la Révolution française et pour réorienter ce sentiment contre l'esclavage en Amérique.

Thomas Branagan, ancien marchand d'esclaves vivant à Philadelphie, élargit la relation entre l'esclavage et le jacobinisme dans ses nombreux textes antiesclavagistes publiés au début des années 1800. Dans son Preliminary Essay (1804), Branagan expliquait qu'il devait utiliser un langage saturé de sang pour attaquer l'esclavage parce que « à mes efforts sérieux, l'oreille est fermée $»^{53}$. La raison objective ne pouvait retourner

(52) Amynto, Reflections on the Inconsistency of Man, Particularly Exemplified in the Practice of Slavery in the United States, New York, John Buell, 1796.

(53) Thomas Branagan, A Preliminary Essay, on the Oppression of the Exiled Sons of Africa Consisting of Animadversions on the Impolicy and Barbarity of the Deleterious Commerce 
la population contre l'esclavage mais l'anti-jacobinisme le pouvait. Les écrits de Branagan établirent de nombreux rapports entre la brutalité de l'esclavage et la violence jacobine. Branagan prévenait que Dieu punirait les États-Unis pour le crime de l'esclavage en infligeant au pays une invasion française. « Vous n'avez pas été réduits à la nécessité douloureuse de voir vos enfants fracassés contre les pierres, ou transpercés par la pointe de la baïonnette, pas plus que vous ne pouvez vous représenter l'image d'un parent bien-aimé mis en pièces par un boulet sorti de la gueule d'un canon, brûlé vif, ou accroché à un gibet », écrivait Branagan, se référant aux horreurs décrites dans les récits anti-jacobins sur les guerres menées par l'armée des républicains français contre les États voisins. Branagan énonçait la mise en garde que, si les Américains poursuivaient sur la voie de l'esclavage, l'armée française pourrait envahir New York et enlever dix mille personnes qui deviendraient esclaves aux Antilles ${ }^{54}$. Ou si Dieu ne châtiait pas les États-Unis par la violence entre les mains des Français eux-mêmes, alors les jacobins du Sud esclavagistes détruiraient la nation. Branagan dépeignait l'institution de l'esclavage comme une institution décivilisatrice qui enlevait son sens moral à l'homme et menait inévitablement à l'effondrement de la société et à l'anarchie. Les propriétaires d'esclaves étaient « des hommes cruels, ambitieux, intéressés, qui traverseraient des mers de sang de leurs frères humains, afin de s'élever euxmêmes ». Les maîtres d'esclaves n'avaient aucun sens de la justice; ils n'étaient que des loups, et s'ils se trouvaient à la tête des États-Unis, la nation serait « déchirée en morceaux », comme la République française ${ }^{55}$.

La mise en garde de Branagan selon laquelle la France menaçait d'envahir les États-Unis effraya sans doute certains lecteurs mais les conservateurs du Nord-Est jugeaient bien plus terrifiant le danger que les États-Unis puissent s'unir à l'équipe «jacobine» de Napoléon. Lorsque le président Madison, démocrate de Virginie, déclara la guerre à

and Subsequent Slavery of the Human Species To Which Is Added, a Desultory Letter Written to Napoleon Bonaparte, Anno Domini 1801, Philadelphia, imprimé pour l'auteur par J.W. Scott, 1804, p. 219 , p. 169 , p. 186.

(54) Thomas Branagan, A Preliminary Essay... op. cit., p. 170, p. 186; Id., The Penitential Tyrant, a Juvenile Poem, in Two Cantos To Which Is Prefixed, Compendious Memoirs of the Author, Philadelphia, imprimé pour l'auteur, 1805, p. 227.

(55) Id., Avenia, or, a Tragical Poem on the Oppression of the Human Species and Infringement on the Rights of Man, Philadelphia, imprimé pour Silas Engles et Samuel Wood, New York, 1805, p. 311-312; Id., Serious Remonstrances, Addressed to the Citizens of the Northern States, and Their Representatives Being an Appeal to Their Natural Feelings \& Common Sense Consisting of Speculations and Animadversions on the Recent Revival of the Slave Trade in the American Republic, Philadelphia, imprimé et publié par Thomas T. Stiles, 1805. 
la Grande-Bretagne en 1812, faisant des États-Unis les alliés implicites de la France napoléonienne, tous les Fédéralistes au Congrès et au Sénat votèrent contre la déclaration de guerre. Les Fédéralistes eurent recours à un langage anti-jacobin pour expliquer leur opposition. Le Fédéraliste du Vermont John Wallace émit la critique que Madison avait fabriqué une «Alliance Française » qui donnerait naissance à un système de "guillotines » et de « meurtres à minuit » dans le pays ${ }^{56}$. Une convention de politiciens opposés à la guerre dans le New Jersey incrimina la guerre en ce qu'elle établissait une « relation avec la France » qui laisserait les ÉtatsUnis en proie à la brutalité et à la ruine ${ }^{57}$.

Les opposants à la guerre associèrent leurs critiques anti-jacobines à un langage antiesclavagiste plein de passion. Elijah Parish, qui avait d'abord prêché contre la Révolution française en 1792, mena la charge antiesclavagiste contre la guerre de 1812 . Le message de guerre du président Madison en 1812 justifiait le conflit en partie pour faire valoir les droits des marins enrôlés de force par la Grande-Bretagne. Si Madison était si humain, d'après Parish, " pourquoi continue-t-il à manier le fouet de l'oppression à l'égard des esclaves qui noircissent ses champs $\|^{58}$ ? Dans un autre sermon contre la guerre, Parish mit les démocrates du Sud en fureur en appelant les hommes du Nord à se rassembler afin de s'opposer au recrutement et de laisser les habitants du Sud en supporter les conséquences. "Que vos seigneurs puissants se satisfassent de faire subir le fouet sanglant à plus d'un million d'esclaves africains » ainsi que l'exprimait Parish. Par son utilisation de l'adjectif français "puissant» pour évoquer le parti de la guerre démocrate, Parish unissait les sentiments anti-jacobin, antiesclavagiste et anti-belliciste, pour en faire un discours d'attaque énergique ${ }^{59}$. L'anti-jacobinisme et l'antiesclavagisme étaient devenus des éléments qui se renforçaient l'un l'autre dans la rhétorique du Nord-Est, et l'une de ces accusations provoquait souvent l'autre.

L'opposition des Fédéralistes ne mit pas fin à la guerre mais la guerre de 1812 vit bien la fin des Fédéralistes. Après le traité de Gand et la victoire triomphale d'Andrew Jackson à la bataille de la Nouvelle-Orléans,

(56) John Wallace, An Oration, Delivered before the Washington Benevolent Society in Newbury, Vermont, on the Fourth of July, 1812, Windsor Bt., Thomas M. Pomroy, 1812.

(57) Federal Party (N.J.), Proceedings and Address of the Convention of Delegates to the People of New Jersey, Trenton, N.J., 1812, p. 8-10.

(58) Elijah PARish, A Discourse, Delivered at Byfield, on the Annual Fast, April 8, 1813, Portland Me., Arthur Shirley, 1813, p. 13.

(59) Id., A Sermon Preached at Byfield, on the Annual Fast, April 11, 1811, 3e éd., Newburyport Mass., E.W. Allen, 1811, p. 16-17. 
les Fédéralistes furent discrédités en tant que traitres qui avaient cherché à conclure une paix séparée avec la Grande-Bretagne (il y avait du vrai dans cette incrimination). Déjà en position affaiblie avant la guerre, les Fédéralistes perdirent tout pouvoir politique national après celle-ci. Pourtant, ce conflit renforça et amplifia le discours antiesclavagiste anti-jacobin dans la culture politique américaine. Lorsque le mouvement contre l'esclavage reprit vigueur dans le milieu des années 1820, les abolitionnistes tablèrent souvent sur cet anti-jacobinisme particulier au pays pour attaquer le Sud.

L'abolitionniste le plus connu de la période antérieure à la guerre de Sécession, William Lloyd Garrison, aida à établir le rapprochement entre l'antiesclavagisme anti-jacobin des débuts de la période nationale et l'abolitionnisme immédiatiste de la période précédant la guerre. Né en 1805 à Newburyport (Massachusetts) près de Byfield, le village d'Elijah Parish, Garrison se plaça lui-même en apprentissage, à l'âge de treize ans, chez Ephraïm Allen, rédacteur en chef du journal fédéraliste local, The Newburyport Herald. Dans le cadre de son travail, Garrison lut les sermons d'Elijah Parish (imprimés par Allen), les discours de Fisher Ames et des numéros d'archives de l'Herald, qui étaient truffés de langage anti-jacobin ${ }^{60}$. Lorsque Garrison commença à imprimer son journal abolitionniste le Liberator en 1831, il puisa fréquemment dans les leçons linguistiques de son enfance. Le Liberator eut recours à une imagerie anti-jacobine pour se porter à l'assaut de l'esclavage, des propriétaires d'esclaves et des anti-abolitionnistes. Le «jacobinisme» de ceux qui étaient favorables à l'esclavage, d'après Garrison, venait directement du 《 système maudit de l'esclavage $\|^{61}$. L'esclavage engendrait de la violence non seulement dans la zone où il existait mais aussi dans les États libres. Le jacobinisme, pour Garrison, exaltait les nordistes pro-sudistes qui, pleins de haine, attaquaient les abolitionnistes. À la suite des violences dans les rues contre l'abolition en 1835, Garrison fit la mise en garde que « les scènes de la Révolution française se reproduiraient, et hommes, femmes, et même enfants, seraient mis à mort par des bouchers humains jusqu'à ce que la terre soit saturée de sang ${ }^{62}$. Son image d'une terre détrempée par le sang répondait exactement comme un écho au sermon d'Elijah Parish de 1794, dans lequel il décrivait la France comme un « sol

(60) Marc Arkin, « The Federalist Trope Power and Passion in Abolitionist Rhetoric », Journal of American History 88, $\mathrm{n}^{\circ}$ 1, 2001, Henry MAYER, All on Fire William Lloyd Garrison and the Abolition of Slavery, New York, St. Martin's Press, 1998.

(61) «The Reign of Terror », dans Liberator, du 15 août 1835.

(62) « Boston Saturday, November 7, 1835 », dans Liberator, du 7 novembre 1835. 
inondé de sang » et avertissait que les Jacobins français amèneraient une «pluie de sang » qui viendrait « rougir nos champs ». Comme Parish, Garrison opérait une synthèse entre l'opposition à l'esclavage et l'antijacobinisme, laquelle devenait un discours d'attaque.

De nombreux abolitionnistes rejoignirent Garrison pour décrire l'esclavage dans le Sud comme un «régime de terreur ». Certains suivirent son exemple, d'autres arrivèrent au même point dans leur argumentation de manière indépendante. Un pasteur du Massachusetts, William Ellery Channing, comme Garrison, établit le lien entre le mouvement anti-jacobin originel et l'anti-esclavage d'avant la guerre. Dans sa jeunesse, Channing avait attaqué la violence de la France révolutionnaire et s'était vivement opposé à la participation des Américains à la guerre de $1812^{63}$. Après la guerre, Channing fit de plus en plus porter son attention sur la question de l'esclavage. À la fin de sa vie, il lia les deux fils conducteurs de sa carrière. Dans son dernier discours public, en 1841, Channing évoqua le « régime de terreur et de force » qu'était la plantation du sud ${ }^{64}$. D'autres réitérèrent son accusation. Une allocution abolitionniste publiée par l' « American and Foreign Anti-Slavery Society » en 1843 faisait mention de ce que les propriétaires d'esclaves avaient «établi un régime de terreur aussi insurrectionnel et sanguinaire dans son principe que celui mis en place par les sans-culottes de la Révolution française $»^{65}$. La presse utilisait le même langage. Le Provincial Freeman, journal canadien publié par des esclaves qui s'étaient enfuis des États-Unis, qualifiait les événements dans le Sud esclavagiste de "si étranges, si contraires à la nature, si monstrueux, si indescriptibles, si grotesques » qu'ils ressemblaient au « régime de la terreur dans la Révolution française $»^{66}$. Il n'était pas nécessaire d'avoir été élevé au cœur de la Nouvelle-Angleterre fédéraliste pour découvrir dans l'anti-jacobinisme un discours abolitionniste convaincant.

$\mathrm{Au}$ fur et à mesure que le sentiment abolitionniste se répandit, l'anti-jacobinisme resta un élément fondamental constitutif de son vocabulaire. À la veille de la guerre de Sécession, alors que l'opposition à

(63) William Ellery Channing, A Sermon, Preached in Boston, August 20, 1812, the Day of Humiliation and Prayer, Appointed by the President of the United States, in Consequence of the Declaration of War against Great Britain, Boston, C. Stebbins, 1812.

(64) Id., Emancipation, New York, American Anti-Slavery Society, 1841.

(65) American and Foreign Anti-Slavery Society, Address to the Non-Slaveholders of the South, on the Social and Political Evils of Slavery, New York, S.W. Benedict \& Co., 1843, p. 13.

(66) «Strange and Horrible Features of United States Slavery», dans The Provincial Freeman, du 4 avril 1857. 
l'esclavage était parvenue à un degré de popularité tel qu'il avait contribué à l'élection d'Abraham Lincoln à la présidence, les abolitionnistes continuaient à faire appel au langage de l'anti-jacobinisme pour plaider leur cause. En 1860, Garrison publia un livre, The New « Reign of Terror » in the Slaveholding States ${ }^{67}$, dans lequel il passait en revue la violence de la politique du Sud au cours de l'année précédente. Il ne fut pas étonnant que, lorsque le Sud fit sécession, le New York Herald accusât les États rebelles d'être aussi "stupides et dénués de remords que les Jacobins ». Les crimes de la Révolution française, ainsi que le président Adams en informa Tocqueville, avaient fait grande impression sur la culture politique américaine. L'anti-jacobinisme avait fourni au Nord-Est un langage pour identifier la menace constituée par la violence du Sud. Désormais, il donnait au Nord-Est un langage pour mettre fin, dans la violence, à cette menace.

\author{
Rachel Hope Cleves \\ Department of History \\ University of Victoria \\ Victoria, B.C. V8W 3P4 \\ Canada \\ rcleves@uvic.ca
}

(67) William Lloyd Garrison, The New «Reign of Terror » In the Slaveholding States, The Anti-Slavery Crusade in America, New York, Arno Press, 1969. 